\title{
HANDS.DVI: \\ A DeVice-Independent programming and control framework for robotic HANDS
}

\author{
Gionata Salvietti ${ }^{1}$, Guido Gioioso ${ }^{1}$, Monica Malvezzi ${ }^{1}$, Domenico Prattichizzo ${ }^{1}$, \\ Alessandro Serio ${ }^{2}$, Edoardo Farnioli ${ }^{2}$, Marco Gabiccini ${ }^{2}$, Antonio Bicchi ${ }^{2}$, \\ Ioannis Sarakoglou ${ }^{3}$, Nikos Tsagarakis ${ }^{3}$, and Darwin Caldwell ${ }^{3}$ \\ ${ }^{1}$ Dept. of Information Engineering and Mathematics, University of Siena \\ \{salviettigio, gioioso, malvezzi, prattichizzo\}edii.unisi.it \\ ${ }^{2}$ Centro E. Piaggio, University of Pisa \\ \{alessandro.serio, edoardo.farnioli,gabiccini,bicchi\}@ \\ centropiaggio.unipi.it \\ ${ }^{3}$ Dept. of Advanced Robotics, Istituto Italiano di Tecnologia \\ \{ioannis.sarakoglou, nikos.tsagarakis, darwin.caldwell\}@iit.it
}

\begin{abstract}
The scientific goal of HANDS.DVI consists of developing a common framework to programming robotic hands independently from their kinematics, mechanical construction, and sensor equipment complexity. Recent results on the organization of the human hand in grasping and manipulation are the inspiration for this experiment. The reduced set of parameters that we effectively use to control our hands is known in the literature as the set of synergies. The synergistic organization of the human hand is the theoretical foundation of the innovative approach to design a unified framework for robotic hands control. Theoretical tools have been studied to design a suitable mapping function of the control action (decomposed in its elemental action) from a human hand model domain onto the articulated robotic hand co-domain. The developed control framework has been applied on an experimental set up consisting of two robotic hands with dissimilar kinematics grasping an object instrumented with force sensors.
\end{abstract}

Keywords: Robotic hand, grasping, object-based mapping, human hand synergies

\section{Introduction}

The HANDS.DVI experiment deals with the development of a unified structure for programming and controlling robotic hands based on a number of fundamental primitives, and abstracting, to the possible extent, from the specifics of their kinematics, mechanical construction, sensor equipment. HANDS.DVI hinges on the study of how the embodied characteristics of the human hand and its sensors, the sensorimotor transformations, and the many constraints they impose, affect and determine the learning and control strategies we use for such fundamental cognitive functions as exploring, grasping and manipulating. The ultimate goal is to learn how to devise simplified and device 


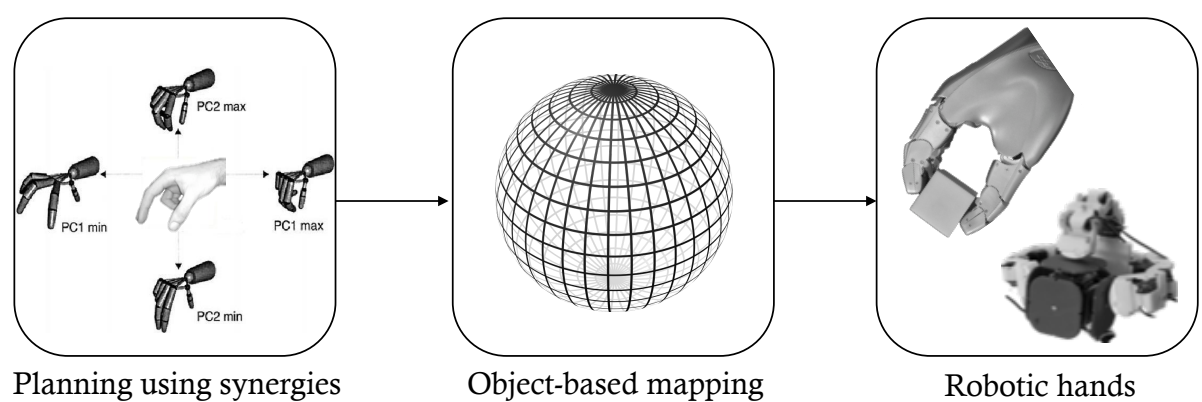

Fig. 1. Idea of the project. The synergies defined for a paradigmatic hand model are used as a simplified language to describe different grasping and manipulation tasks. The arising motion is mapped through and object based approach to different robotic hands without considering the specific kinematics.

independent control system architectures for robotic hands from human data available in the literature and hypotheses driven simulations. The acronym HANDS.DVI was chosen in analogy to device-independent files that are generated by the TeX typesetting language.

The experiment has been separated into three different phases called SYN, DVI and EXP. The phase SYN dealt with the development of strategies for grasp force control based on studies in neuroscience concerning the sensorimotor organization of the human hand [1]. These studies demonstrated that, notwithstanding the complexity of the hand, a few variables are able to account for most of the variance in the patterns of configurations and movements. The reduced set of parameters that humans effectively use to control their hands, known in the literature as synergies, represents a possible set of words for a unified control language for robotic hands. In the phase DVI, we focused on designing control algorithms based on synergies for robotic hands with a generic kinematic structure, not necessarily bio-inspired. We developed on object-based approach to map human synergies onto several robotic hand types including non-anthropomorphic hands. The main idea of the mapping algorithm was to involve a virtual object as a mediator between human and robotic hand. Finally, in phase EXP, we implemented and evaluated the synergy based approach on two devices: the ModHa 39p hand, a modular hand developed at University of Siena, and the DLR-HIT II hand. For the evaluation we also used an instrumented object developed within the experiment that allows to measure contact point positions and the relative contact forces. The general framework of the experiment is pictorially represented in Fig. 1.

The rest of the chapter is organized as it follows. Section 2 deals with the definition of a paradigmatic hand able to capture the synergistic organization of the human hand. In Section 3 a description of the object-based mapping, that represents the algorithmic core of HANDS.DVI, is given. In Section 4 the setup and relative results for the experiments in motion and force control evaluation are shown. Finally in Section 5 conclusions and future possible applications are outlined. 


\section{The paradigmatic hand}

A deeper understanding of the way humans use their hands enable an approach to programming hands that allows users to more easily control the different devices that may be used in a robotic system, by encapsulating the hand hardware in functional modules, and ignoring the implementation-specific details. In the HANDS.DVI experiment, such new methodology for grasping analysis has been based on the concept of synergies. The results on the organization of the human hand in grasping and manipulation presented in $[1,2]$ were based on experimental tests in which subjects were asked to perform grasping actions on a wide variety of objects. Data were recorded by means of data gloves and were analysed with principal component analysis (PCA) techniques. The results showed that the first two principal components account for most of the variability in the data, more than $80 \%$ of the variance in the hand postures. In this context the principal components were referred to as synergies to capture the concept that, in the sensorimotor system of the human hand, combined actions are favoured over individual component actions, with advantages in terms of simplification and efficiency of the overall system. Further, synergies where shown to exist not only in movement of hand configurations while preparing to grasp but also in force control. In [2] the authors suggested an explanation of the coordination of isometric forces exerted during grasping, namely that there exist a few basic patterns, the synergies, which are suitable for coarse control of force and that these synergies can be modified by superimposing a finer control. To summarize the results of the recent neuroscience studies: notwithstanding the human hand is characterized by a complex mechanical structure with many degrees of freedoms (DoFs) it results that most of the actions of the human hand can be represented as a combination of a relatively small number of basic primitives of motion, referred to as synergies. In other words, even if the human hand has a complex kinematic structure, with many degrees of freedom, in most everyday tasks it appears to be controlled by a much smaller set of knobs, corresponding to compound actions, movements and variables. Our idea is to use this few knobs to control different robotic hands without considering the specific kinematic. However the development of such control framework passes through the definition of a generic human hand model, i.e. a paradigmatic hand, where the synergies can be easily defined.

The paradigmatic hand is a kinematic model inspired by the human hand that represents a trade-off between the complexity of the human hand model accounting for the synergistic organization of the sensorimotor system and the simplicity, and accessibility, of the models of robotic hands available on the market. Examples of human hand biomechanical models are available in the literature $[3,4]$. The fingers are usually modelled as kinematic chains independent from each other, sharing only their origin in the hand palm. In absence of disabilities or handicaps, the ratios between the bones lengths of each finger are almost constant [5]. Hence, in Tab. 1 the bones length ratios defined with respect to the length of the distal phalanges bone (dp) of each finger [6] are reported (for bone acronyms please refer to the table caption). 
Table 1. Table of bone-to-bone length ratios (Bone names: distal phalanx (dp), proximal phalanx (pp), middle phalanx (mp), metacarpal (mc)).

\begin{tabular}{|c|c|c|c|c|}
\hline Finger & & $m p / d p$ & $p p / d p$ & $m c / d p$ \\
\hline Thumb & right & - & 1.37 & 2.09 \\
\hline & left & - & 1.36 & 2.08 \\
\hline Index & right & 1.41 & 2.45 & 4.17 \\
\hline & left & 1.41 & 2.44 & 4.10 \\
\hline Middle & right & 1.60 & 2.54 & 3.71 \\
\hline & left & 1.59 & 2.54 & 3.71 \\
\hline Ring & right & 1.50 & 2.33 & 3.25 \\
\hline & left & 1.49 & 2.31 & 3.22 \\
\hline Pinky & right & 1.15 & 2.04 & 3.32 \\
\hline & left & 1.16 & 2.04 & 3.32 \\
\hline
\end{tabular}

The human hand joints can mainly be divided into 1-DoF and 2-DoF joints. The 1-DoF joints in the hand can be represented as revolute joints; the 2-DoF joints can be divided into two types. The trapeziometacarpal joint of the thumb is a saddle joint with non-orthonormal axes, the metacarpophalangeal joints of the fingers are condyloid. The main difference between saddle and condyloid joints is that condyloid joints have approximately intersecting axes while saddle joints do not. For the thumb, the axes of the metacarpal are non-orthogonal screw. Therefore, the metacarpophalangeal joint of the index, middle, ring and little fingers are usually modelled as a two DoFs joint (one for adduction/abduction and another flexion/extension). The proximal interphalangeal and distal interphalangeal joints of the other fingers can be modelled as a one DoF (revolute) joint. The thumb has at least 5 DoF: 2 DoF in trapeziometacarpal joint, 2 DoF in metacarpophalangeal joint, and $1 \mathrm{DoF}$ in interphalangeal joint. Anyway, the range of deviation of metacarpophalangeal joint is so small that generally can be modelled as
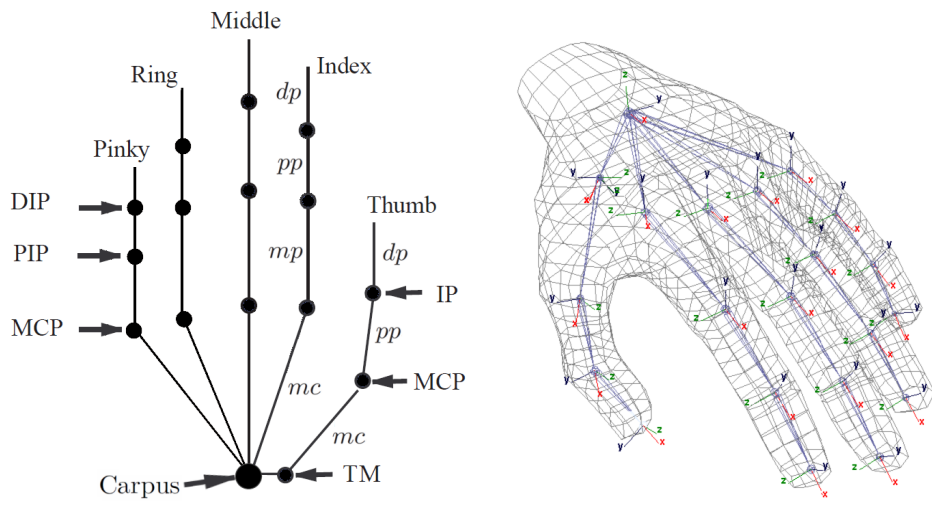

Fig. 2. 20 DoFs kinematic model of the paradigmatic hand. 
Table 2. Allowed ranges for the joint angle variables.

\begin{tabular}{|c|c|c|c|c|}
\hline Finger & $q_{1}$ & $q_{2}$ & $q_{3}$ & $q_{4}$ \\
\hline Thumb & $-10^{\circ}, 80^{\circ}$ & $0^{\circ},-55^{\circ}$ & $0^{\circ},-55^{\circ}$ & $0^{\circ},-40^{\circ}$ \\
\hline Index & $0^{\circ}, 90^{\circ}$ & $-15^{\circ}, 15^{\circ}$ & $0^{\circ}, 110^{\circ}$ & $0^{\circ}, 90^{\circ}$ \\
\hline Middle & $0^{o}, 90^{\circ}$ & $-12^{\circ}, 12^{\circ}$ & $0^{\circ}, 110^{\circ}$ & $0^{\circ}, 90^{\circ}$ \\
\hline Ring & $0^{\circ}, 90^{\circ}$ & $-10^{\circ}, 10^{\circ}$ & $0^{o}, 110^{\circ}$ & $0^{\circ}, 90^{\circ}$ \\
\hline Pinky & $0^{\circ}, 90^{\circ}$ & $-12^{\circ}, 12^{\circ}$ & $0^{\circ}, 110^{\circ}$ & $0^{\circ}, 90^{\circ}$ \\
\hline
\end{tabular}

a single DoF joint, while the trapeziometacarpal joint is more important in the analysis of the thumb kinematics [5]. In order to avoid unnatural finger positions, the set of angle constraints reported in Tab. 2 is taken into account [7]. Our model, showed in Fig.2, has therefore 20 DoFs corresponding to 4 DoFs for the thumb (TR, TA, TM, TI - Thumb Rotation, Abduction, Metacarpal, Interphalangeal) and 4 DoFs for the index, middle, ring and pinky (Index Abduction, Metacarpal, Proximal interphalangeal, Distal interphalangeal).

\section{The mapping algorithm}

In this Section the mapping algorithm developed in the DVI phase is revised. For further details, the reader is referred to [8,9]. The proposed mapping algorithms tries to match the effects of the manipulation tasks performed by the paradigmatic hand, and the real robotic hand. In other terms the paradigmatic hand drives the real robotic hand through this mapping. The proposed approach is not specific for a given task or a given grasped object but can be extended to most of the manipulation tasks. Such a generality is gained considering that the principal actions in manipulation are to guarantee the stability of the grasp and to move the grasped object along planned trajectories. Other mapping methods have been proposed in the literature which generally are based on a joint-tojoint mapping [10] or on a fingertip position mapping [11]. We used a virtual object approach to capture the generality of the many possible objects to be manipulated in our model. Two virtual spheres are used, one for the paradigmatic hand and the other for the robotic hand. These are defined by the hands' posture and change during the task. Note that the use of a spherical virtual object does not restrict the use of this algorithm to spherical objects [8]. The main idea is to reproduce movements and deformations exerted by the paradigmatic human-like hand, controlled by synergies, on the virtual sphere computed as the minimum sphere containing a set of reference points that can be arbitrarily placed on the hands (see Fig. 3).

In the following we describe how the map is obtained. Let the paradigmatic hand be described by the joint variable vector $q_{h} \in \Re^{n_{q h}}$ and assume that the subspace of all configurations can be represented by a lower dimensional input vector $z \in \mathfrak{R}^{n_{z}}$ (with $\left.n_{z} \leq n_{q h}\right)$ which parametrizes the motion of the joint variables along the synergies $q_{h}=$ $S_{h} z$ being $S_{h} \in \mathfrak{R}^{n_{q h} \times n_{z}}$ the synergy matrix. In terms of velocities one gets

$$
\dot{q}_{h}=S_{h} \dot{z} .
$$




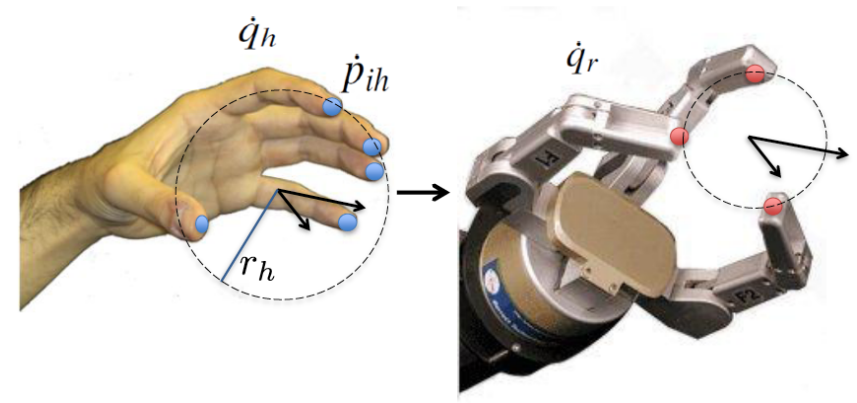

Fig. 3. Mapping synergies from the paradigmatic human hand to the robotic hand: the reference points on the paradigmatic hand $p_{h}$ (blue dots) allows to define the virtual sphere. Activating the human hand synergies, the sphere is moved and strained; its motion and strain can be evaluated from the velocities of the reference points $\dot{p}_{h}$. This motion and strain, scaled by a factor depending on the virtual sphere radii ratio, is then imposed to the virtual sphere relative to the robotic hand, defined on the basis of the reference points $p_{r}$ (red dots).

The ultimate goal of this mapping is to find a way of controlling the reference joint variables $\dot{q}_{r} \in \mathfrak{R}^{n_{q r}}$ of the robotic hand in a synergistic way using the vector of synergies $z$ of the paradigmatic hand. In other terms we want to design a map $S_{r}$ to steer the robotic joint reference variables as follows

$$
\dot{q}_{r}=S_{r} \dot{z}
$$

where map $S_{r}$ depends on the synergy matrix $S_{h}$ and other variables as explained in the following.

To define the mapping we assume that both the paradigmatic and the robotic hands are in given configurations $q_{0 h}$ and $q_{0 r}$ (Fig. 3). A set of reference points $p_{h}$ are chosen on the paradigmatic hand. We have chosen the fingertip points as reference points. Other choices are possible as, for example, in the intermediate phalanges or in the hand palm since the number of reference points can be arbitrary set $[8,9]$.

The virtual sphere object is then computed as the minimum sphere containing the reference points in $p_{h}$ (Fig. 3). Note that these points in general do not lie on the sphere surface. Let us parametrize the virtual sphere by its center $o_{h}$ and radius $r_{h}$. The motion imposed to the hand reference points moves the sphere and changes its radius.

The motion of the hand due to synergies could be described using a large set of parameters, in this algorithm we simplify the problem assuming the following transformation for the virtual sphere:

- a rigid-body motion, defined by the linear and angular velocities of the sphere center $\dot{o}_{h}$ and $\omega_{h}$, respectively

- a non-rigid strain represented by the radius variation $\dot{r}$ of the sphere.

Representing the motion of the hand through the virtual object, the motion of the generic reference point $p_{i h}$ can be expressed as

$$
\dot{p}_{i h}=\dot{o}_{h}+\omega_{h} \times\left(p_{i h}-o_{h}\right)+\dot{r}_{h}\left(p_{i h}-o_{h}\right) .
$$


Grouping all the reference point motions, one gets

$$
\dot{p}_{h}=A_{h}\left[\begin{array}{c}
\dot{o}_{h} \\
\omega_{h} \\
\dot{r}_{h}
\end{array}\right],
$$

where matrix $A_{h} \in \mathfrak{R}^{n_{c h} \times 7}$ is defined as follows

$$
A_{h}=\left[\begin{array}{ccc}
I & -S\left(p_{1 h}-o_{h}\right) & \left(p_{1 h}-o_{h}\right) \\
\cdots & \cdots & \cdots \\
I & -S\left(p_{i h}-o_{h}\right) & \left(p_{i h}-o_{h}\right) \\
\cdots & \cdots & \cdots
\end{array}\right]
$$

and $S()$ is the skew operator. Matrix $A_{h}$ depends on the type of motion that we decide to reproduce on the robotic hand and then it depends on the task. From these equations we can evaluate the virtual sphere motion and deformation as a function of the synergy vector velocity $\dot{z}$ of the paradigmatic hand

$$
\left[\begin{array}{c}
\dot{o}_{h} \\
\omega_{h} \\
\dot{r}_{h}
\end{array}\right]=A_{h}^{\#} \dot{p}_{h}=A_{h}^{\#} J_{h} S_{h} \dot{z},
$$

where $A_{h}^{\#}$ denotes the pseudo-inverse of matrix $A_{h}$. We now need to map these motions and deformations on the robotic hand. The robotic hand is in a given configuration $q_{0 r} \in$ $\Re^{n_{q r}}$ with resulting reference point location vector $p_{r} \in \mathfrak{R}^{n_{c r}}$. Note that no hypothesis were imposed on the number of reference points on the paradigmatic human and robotic hands, in general we can consider $n_{c h} \neq n_{c r}$, neither on their locations, and neither on the initial configuration of the two hands. The same use of the virtual sphere is applied here: find the minimum sphere enclosing the reference points and indicate with $o_{r}$ its center coordinates and with $r_{r}$ its radius (Fig. 3). Let us thus define the virtual object scaling factor as the ratio between the sphere radii $k_{s c}=\frac{r_{r}}{r_{h}}$. This factor is necessary to scale the velocities from the paradigmatic to the robotic hand workspaces. Note that the scaling factor depends on the hand dimensions, but also on their configuration.

Then, the motion and deformation of the virtual sphere generated by the paradigmatic hand are scaled and tracked by the virtual sphere referred to the robotic hand

$$
\left[\begin{array}{c}
\dot{o}_{r} \\
\omega_{r} \\
\dot{r}_{r}
\end{array}\right]=K_{c}\left[\begin{array}{l}
\dot{o}_{h} \\
\omega_{h} \\
\dot{r}_{h}
\end{array}\right]
$$

where the scale matrix $K_{c} \in \Re^{7 \times 7}$ is defined as

$$
K_{c}=\left[\begin{array}{ccc}
k_{s c} I_{3,3} & 0_{3,3} & 0_{3,1} \\
0_{3,3} & I_{3,3} & 0_{3,1} \\
0_{1,3} & 0_{1,3} & 1
\end{array}\right] .
$$

According to eq. (4) and (5), the corresponding robot reference point velocity is given by

$$
\dot{p}_{r}=A_{r}\left[\begin{array}{c}
\dot{o}_{r} \\
\omega_{r} \\
\dot{r}_{r}
\end{array}\right]
$$


where matrix $A_{r} \in \mathfrak{R}^{n_{c r} \times 7}$ is defined as follows

$$
A_{r}=\left[\begin{array}{ccc}
I & -S\left(p_{1 r}-o_{r}\right) & \left(p_{1 r}-o_{r}\right) \\
\cdots & \cdots & \cdots \\
I & -S\left(p_{i r}-o_{r}\right) & \left(p_{i r}-o_{r}\right) \\
\cdots & \cdots & \cdots
\end{array}\right] .
$$

Recalling eq. (6) and (7) we can express the robotic hand reference point velocities $\dot{p}_{r}$ as a function of the synergy velocities $\dot{z}$

$$
\dot{p}_{r}=A_{r} K_{c} A_{h}^{\#} J_{h} S_{h} \dot{z}
$$

and, considering the robot hand differential kinematics $\dot{p}_{r}=J_{r} \dot{q}_{r}$, where $J_{r} \in \mathfrak{R}^{n_{c r} \times n_{q r}}$ is its Jacobian matrix, the following relationship between robot hand joint velocities and synergy velocities is defined

$$
\dot{q}_{r}=J_{r}^{\#} A_{r} K_{c} A_{h}^{\#} J_{h} S_{h} \dot{z} .
$$

Finally the synergy mapping $S_{r}$ in (2) for the robotic hand is computed as

$$
S_{r}=J_{r}^{\#} A_{r} K_{c} A_{h}^{\#} J_{h} S_{h},
$$

where $J_{r}^{\#}$ is the pseudoinverse of the Jacobian of the robotic hand and $J_{h}$ is the Jacobian of the paradigmatic hand. Note that matrix $J_{r}^{\#} A_{r} S_{c} A_{h}^{\#} J_{h}$ depends on

- paradigmatic and robotic hand configurations $q_{0 h}$ and $q_{r h}$;

- location of the reference points for the paradigmatic and robotic hands, $p_{h}$ and $p_{r}$.

\section{Experimental Result}

The object-based mapping able to transfer human hand synergies onto robotic hand has been validated in an experimental setup where the capability of reproducing object motions and exerted grasping forces were considered. All the experiments have been performed on two model of robotic hands: the DLR-HIT II hand [12] and the ModHa39p hand [8]. The DLR-HIT II hand has an anthropomorphic structure with 5 fingers, 15 DoFs and 12 actuated joints. The ModHa39p hand is a fully-actuated robotic hand with a modular structure. Each module $(42 \times 33 \times 16 \mathrm{~mm})$ has one DoF and it can be easily connected to the others obtaining kinematic chains that we can consider as fingers. These chains are connected to a common base that can be thought as a palm. In the proposed configuration each finger has 3 DoFs, thus the hand has globally 9 DoFs. In the rest of the Section, the obtained results are presented.

\subsection{Object motion evaluation}

In this experiment we compared the trajectory of the center of a virtual grasped object moved by the paradigmatic hand and the same trajectory of a real object grasped and moved by the robotic hands. A tracking system was thus necessary. We decided to use a cheap and efficient optical system since that met our specifics on precision. In particular, 


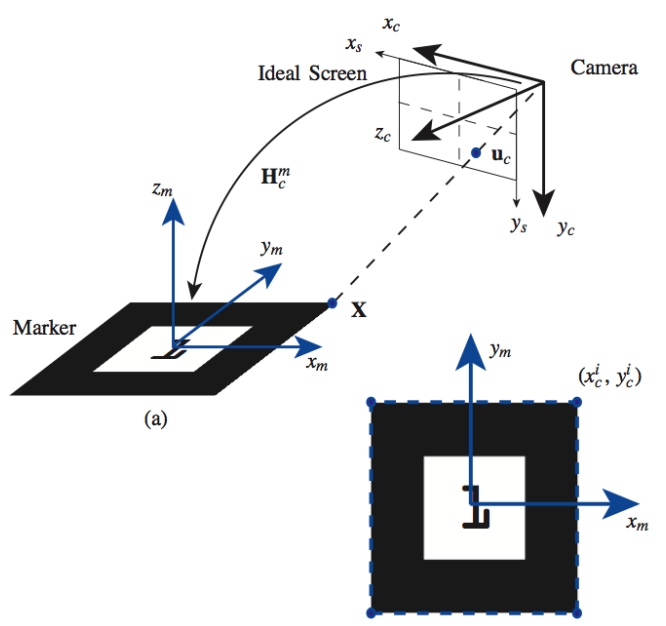

(b)

Fig. 4. ARToolkit coordinates frames: (a) camera frame $\left\langle x_{c}, y_{c}, z_{c}\right\rangle$, marker frame $\left\langle x_{m}, y_{m}, z_{m}\right\rangle$ and ideal screen frame $\left\langle x_{s}, y_{s}\right\rangle$; (b) marker line contour (dotted lines) and corners $\left(x_{c}^{i}, y_{c}^{i}\right), i=1,2,3,4$.

we used the ARToolkit library $[13,14]$ to track the motion of a marker placed on the grasped object through a camera.

ARToolKit is a $\mathrm{C}$ and $\mathrm{C}++$ language software library that lets programmers easily develop Augmented Reality applications. It uses computer vision techniques to compute the real camera position and orientation relative to marked cards, allowing the programmer to overlay virtual objects onto these cards. Given square markers are used as a base of the coordinates frame where virtual objects are represented.

Let us consider the setup reported in Fig. 4 where a perspective camera $\langle c\rangle$ is observing a marker $\langle m\rangle$. If we call the 3 -D coordinates of a point $\mathbf{X}$ relative to the camera and marker reference frame $\mathbf{P}_{c} \in \mathbf{R}^{3}$ and $\mathbf{P}_{m} \in \mathbf{R}^{3}$, they are related by a rigid-body transformation

$$
\mathbf{P}_{c}=\mathbf{R}_{c}^{m} \mathbf{P}_{m}+\mathbf{t}_{c}^{m},
$$

where $\mathbf{R}_{c}^{m} \in \mathbf{S} O(3)$ is the rotation matrix which relates the camera and marker reference frame and $\mathbf{t}_{c}^{m}$ is the corresponding translation vector. Considering $\widetilde{\mathbf{P}}_{c}, \widetilde{\mathbf{P}}_{m}$ the relative extension in homogeneous coordinates, eq. (14) can be written as

$$
\widetilde{\mathbf{P}}_{c}=\mathbf{H}_{c}^{m} \widetilde{\mathbf{P}}_{m}
$$

where

$$
\mathbf{H}_{c}^{m}=\left[\begin{array}{cc}
\mathbf{R}_{c}^{m} & \mathbf{t}_{c}^{m} \\
\mathbf{0}_{1 \times 3} & 1
\end{array}\right] .
$$




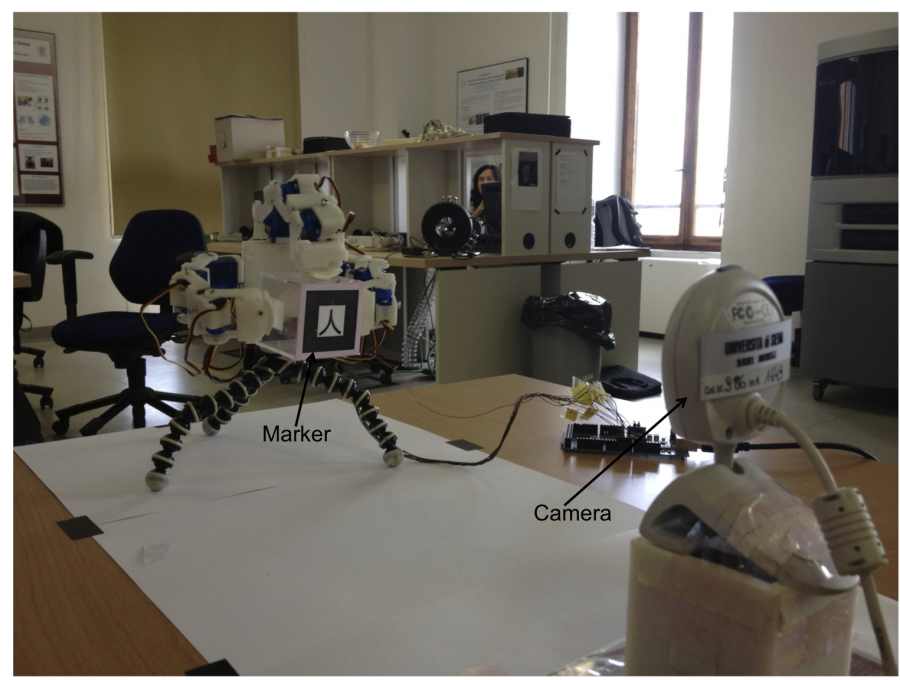

Fig. 5. The experiment setup for object motion evaluation. The camera measures the movement of the marker placed on the grasped object.

Let us assume that the intrinsic camera calibration matrix is given by,

$$
\mathbf{K}=\left[\begin{array}{ccc}
f_{x} & s & u_{0} \\
0 & f_{y} & v_{0} \\
0 & 0 & 1
\end{array}\right]
$$

where $f_{x}, f_{y}$ (pixels) denote the focal lengths of the camera along the $\mathrm{x}$ and $\mathrm{y}$ directions, $\mathrm{s}$ is the skew factor and $\left(u_{0}, v_{0}\right)$ (pixels) is the principal point of the CCD. According to perspective projection models, the projection $\widetilde{\mathbf{u}}_{c} \triangleq\left[x_{c} y_{c} 1\right]^{T}$ of $\widetilde{\mathbf{P}}_{c}$ on the camera is given by

$$
\widetilde{\mathbf{u}}_{c}=\mathbf{K}\left[\begin{array}{ll}
\mathbf{I} & 0
\end{array}\right] \widetilde{\mathbf{P}}_{c},
$$

where $\mathbf{I} \in \mathbf{R}^{3 \times 3}$ represents the identity matrix. By putting together eq. (15) and eq. (17) we obtain,

$$
\widetilde{\mathbf{u}}_{c}=\mathbf{K}[\mathbf{I} \mathbf{0}] \mathbf{H}_{c}^{m} \widetilde{\mathbf{P}}_{m}
$$

which describes the projection on the camera image plane of a 3-D point expressed in the marker reference frame $\langle m\rangle$. The marker pose and position $\left(\mathbf{H}_{c}^{m}\right)$ can be obtained by minimizing the reprojection error

$$
\text { err }=\frac{1}{4} \sum_{i=1,2,3,4}\left(\hat{x}_{c}^{i}-x_{c}^{i}\right)^{2}+\left(\hat{y}_{c}^{i}-y_{c}^{i}\right)^{2}
$$

where $\hat{x}_{c}^{i}, \hat{y}_{c}^{i}$ are noisy measurements of the $i$-th marker corner and $x_{c}^{i}, y_{c}^{i}$ are the ideal corresponding points (see Fig. 4), [15, ch.6], [16, ch.4]. 


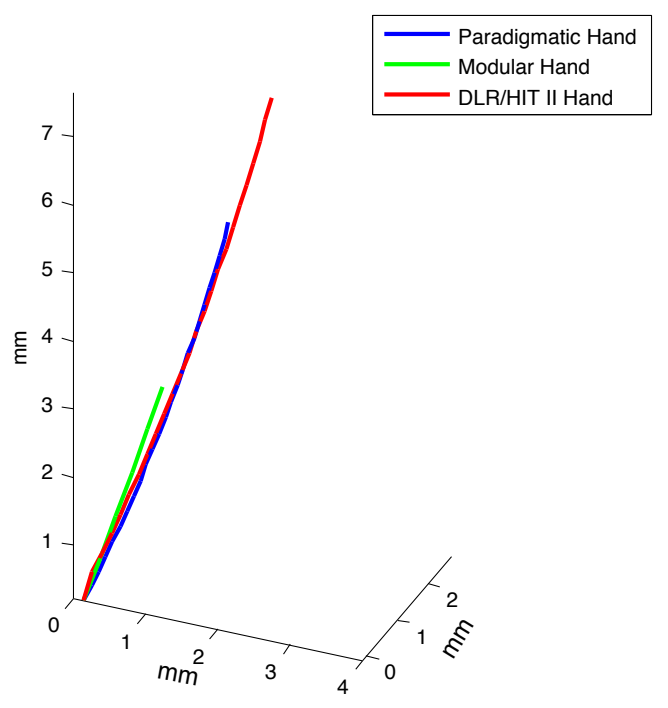

Fig. 6. Object trajectories obtained during the experiments.

In Fig. 5 the setup of the experiment is showed. We used the obtained measures to estimate the motion of the grasped object. We considered a cube as grasped object. Only the first four synergies were activated on the paradigmatic hand model. Given this underactuation condition, for each configuration of the hand, only one feasible rigid body motion of the cube exists, corresponding to a particular combination of the four synergies [17]. This particular combination were activated on the hand at each time step, resulting in an object movement that is represented by the blue line in Fig. 6 .

The synergistic movement of the paradigmatic hand were mapped on the two considered robotic hands. The mapped movement produced an object displacement in the two cases and the resulting trajectories are represented in Fig. 6. We performed 20 trials for each robotic hand and the plotted trajectories that we considered to analyse the performances have been computed as the average of the 20 obtained trajectories. We can observe that the three paths have different lengths. This is due to the scaling factor that we introduced in the mapping algorithm. In particular, the paradigmatic hand performed a $5.9 \mathrm{~mm}$ movement. The ModHa39p performed a $3.61 \mathrm{~mm}$ movement corresponding to the scaling factor 0.61 computed by the mapping algorithm. The DLR-HIT II Hand, with a scaling factor of 1.3, produced an object displacement of $7.69 \mathrm{~mm}$.

Note that the DLR-HIT II hand obtained better results in terms of object motion trajectory. This is due to its higher redundancy and thus dexterity. It was not possible for the ModHa39p to reproduce the movement, given its simple kinematic structure. However the mapping algorithm, with its pseudoinverse computation, produced the closest feasible trajectory for this hand. 


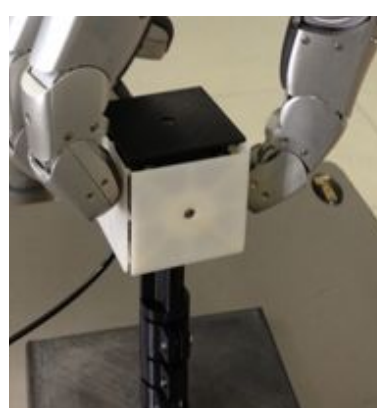

(a)

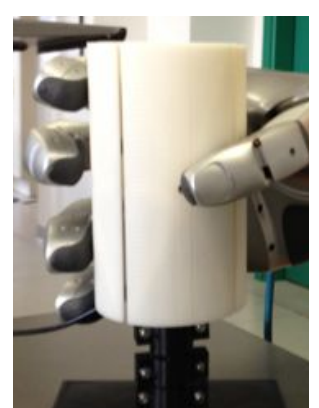

(b)

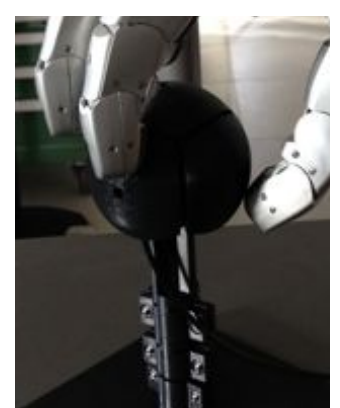

(c)

Fig. 7. The instrumented object: in the cube (a), the cylinder (b) and the sphere (c) configuration.

In the two cases we computed an average error that is the average angular distance between the linear velocity vector of the paradigmatic and the robotic hand at each time step. For the ModHa39p this error was 7.4 degrees while for the DLR-HIT II hand it was practically zero.

\subsection{Internal forces evaluation}

Within the project we developed an instrumented object in order to measure the forces exerted by the robotic hands during grasping and manipulation and to compare them with those exerted by the paradigmatic hand. The instrumented core of the object is a cube with each of its faces being a 6 axis force torque sensor. An external interaction face is mounted at the centre of each face. The external faces are interchangeable and by attaching the appropriate face it is possible to create a cube, a sphere and a cylinder, as shown in Fig. 7. Mounting of the external faces is easy with a single locating screw per face. Therefore change between different objects shapes are relatively fast. With the current size of the instrumented core the smallest object size that can be assembled are:

- a cube of $50 \mathrm{~mm}$ side;

- a sphere of $70 \mathrm{~mm}$ diameter;

- a cylinder of $70 \mathrm{~mm}$ diameter and $120 \mathrm{~mm}$ length.

The size of the object can be increased by attaching larger external faces while respecting the sensing element torque limits.

All electronics and sensitive parts are covered inside the instrumented core. A single flexible cable provides power to the electronics and a fast TCP/IP-UDP connection. The main board uses a Texas LM3S8962 Microcontroller running at 50MHz. The system uses a 16bit DACs to read 36 multiplexed channels. The circuit also uses a 16bit DAC to eliminate the offsets between the channels. The Ethernet is a $100 \mathrm{Mb} / \mathrm{s}$ link which allows a frame rate of $1 \mathrm{kHz}$.

The force/torque elements have been designed based on a FEM analysis in order to ensure a reasonable loading range of the material. For the intended loads material 
stress must be kept low enough to lie within the elastic region of the material. At the same time the strain induced on the measuring gauges should be large enough to allow appropriately low amplification gains ensuring a high signal to noise ratio. In this way fatigue free operation of the material can guarantee long life and repeatable measurement, while high signal to noise ratio should enable high force/torque resolution. To achieve a good signal to noise ratio the amplifier gain has been specified to be in the region of 20. The maximum continuous load (pure force in the middle of the face with no torques) was specified at $100 \mathrm{~N}$. The chosen sensing material was 39NICRMO3 (steel) with a Yield stress between 600-900 MPa. Based on the above specifications a FEM and an electronics analysis was conducted leading to a design with the following predicted characteristics.

For the maximum continuous load the stress of the material was designed to lie approximately in the $1 / 3 \times$ Yield stress range where plastic deformation and fatigue are avoided. Based on the selected gain of 20 the 16Bit DAC provides a force resolution of $3 \mathrm{mN}$ and a force range of $\pm 100 \mathrm{~N}$. The peak load (pure force in the middle of the face with no torques) for a $2 / 3 \times$ Yield-Stress is in the region of $\pm 200 \mathrm{~N}$. This load should not be exceeded to avoid any possibility of plastic deformation and fatigue. The operating range is near $\pm 100 \mathrm{~N}$ for pure force (pure force in the middle of the face with no torques). The sensor has not been designed for continuous cyclic loading. Cyclic loading should be kept sufficiently lower than the maximum continuous load so as to avoid fatigue.

This instrumented object allows 6 wrench vectors ( 1 per external face) to be measured. This limits interactions to one contact point per face. Assuming accurate readings in all $6 \mathrm{DoF}$ (forces $x y z$ and torques $x y z$ ) an arbitrary force vector applied on an external face could be resolved, Fig. 8. A soft finger model at the contact points is computed using the 6 measures and the intrinsic contact sensing algorithm presented in [18].

Another important aspect is that the object can be grounded by mounting one of its faces on a table or other grounded surfaces. This allows experiments with the hand/arm control while a total force is needed to be applied to the environment through the particular object geometry. The grounded object would directly provide forces and torques with respect to the world frame measured at the grounded face as well as those at the robot interaction points.

In order to evaluate the performances of the mapping algorithm in grasping and manipulation tasks we focused on the computation of exerted internal forces. Internal forces are those forces that do not move the contact points playing an important role in the grasp stability [19]. We performed experiments taking advantage of the instrumented object previously described. For the sake of simplicity, only results obtained with the cubic and the spherical configuration of the object are reported in this chapter. The target of the experiment was to compare the internal forces exerted by the paradigmatic hand on the object in simulation environment with those reproduced by the robotic hand on the real instrumented object. A direct comparison between the two hands was not possible due to the fact that the "driven" robotic hand could have a different number of contact points (for instance, the ModHa39p hand can have maximum 3 contact points if fingertip grasping is considered). For this reason, we adopted a measure of the whole object deformation energy produced by the activation of a combi- 


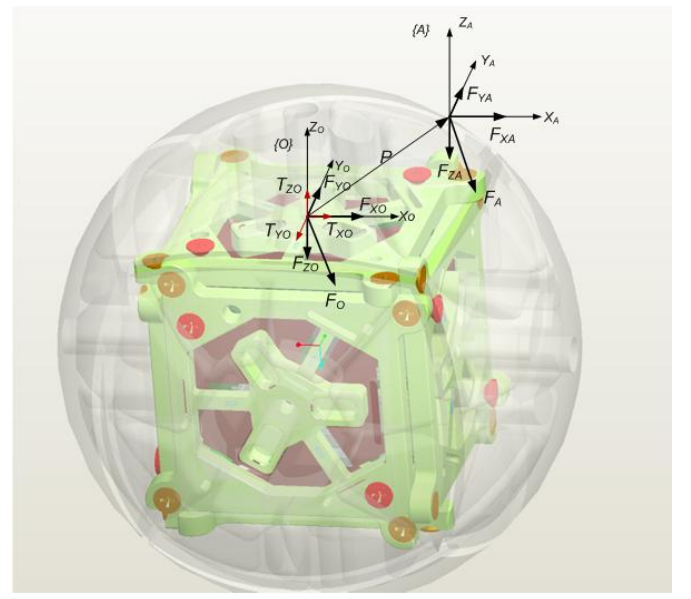

Fig. 8. The vector of a single force $F_{A}$ acting on a surface can be solved from the force and torque vectors measured at the centre of the respective load cell.

nation of synergies to evaluate and compare the performance of the analysed mapping procedures. A variation of the internal forces $\delta \lambda$ is obtained activating a combination of synergies on the paradigmatic hand.

Considering the model of soft synergies described in [17], the contact force variation can be computed considering the associated compliance. Let indicate with $\delta x$ the vector containing the deformation components of each contact point evaluated as $\delta x=K_{s}^{-1} \delta \lambda$. The elastic energy variation produced by the activation of synergies can be computed as

$$
\delta E_{e l}=\frac{1}{2} K_{s}\left\|(\delta x)^{2}\right\|=\frac{1}{2} K_{s}^{-1}\left\|(\delta \lambda)^{2}\right\|,
$$

where $K_{s}$ is a contact stiffness matrix. The $\delta \lambda$ values were computed in simulation environment for the paradigmatic hand while they were read directly from the force sensors of the object for the robotic hands (see Fig. 9). We considered the same $K_{s}$ value for the two hands. We computed the energy variation index expressed in (19) considering the activation of the first three synergies and we evaluated the percentage difference between the paradigmatic and the robotic hand.

The exerted forces and the consequent energy variations due to the synergy activations for the paradigmatic hand were evaluated in simulation environment using the Matlab SynGrasp Toolbox [20]. In Fig. 10 is represented the Matlab model of the paradigmatic hand used for the simulations.

Values obtained in simulation activating separately the first three synergies were compared with energy variations obtained by the robotic hands. The object was kept in a fixed reference position. This allows to compensate the gravity effects. A specific 


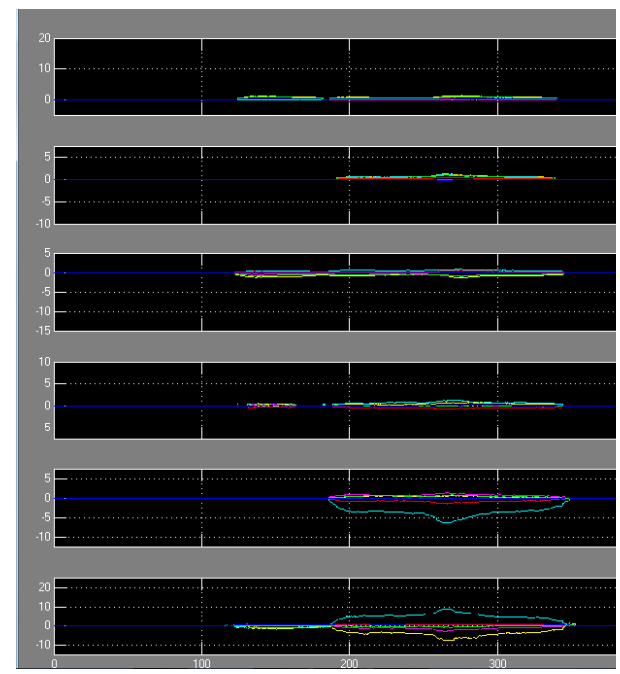

Fig. 9. A screenshot of the sensorized object interface. The six boxes represent, starting from the top, the three forces and the three torques for a single face.

calibration procedure has been developed for this porpoise. In Fig. 11 the two robotic hands grasping the instrumented object in "cube-configuration" are shown, while in Fig. 12 the DLR-HIT II hand grasping the object in "sphere-configuration" is reported.

We performed 20 trials with both the two robotic hands and the two object configuration and we computed the average percentage error obtained by the robotic hands with respect to the total amount of energy variation produced by the paradigmatic hand. Results are summarized in Tables 3 and 4.

Table 3. Energy variation error for the ModHa 39p Hand - Cubic and spherical object

\begin{tabular}{|l|c|c|}
\hline \hline Synergies & \% Error Cubic Obj. & \% \\
\hline \hline Syn 1 & $21 \%$ & $18 \%$ \\
Syn 2 & $10 \%$ & $5 \%$ \\
Syn 3 & $50 \%$ & $45 \%$ \\
\hline \hline
\end{tabular}

It is worth noting that, as expected, the DLR-HIT II hand achieves best performances also in terms of energy. The worst performances that we obtained correspond to the third synergy mapped on the ModHa 39p. This can be explained considering that the third synergy is substantially a movement that constrains the fingers of the paradigmatic hand to spread out mainly using the adduction/abduction joints. This kind of movement 


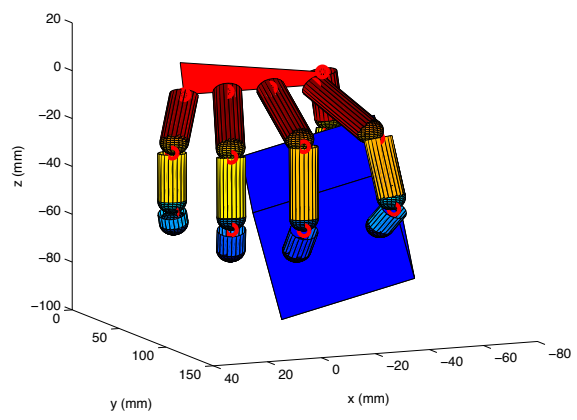

Fig. 10. The Paradigmatic Hand model grasping the cube in SynGrasp.
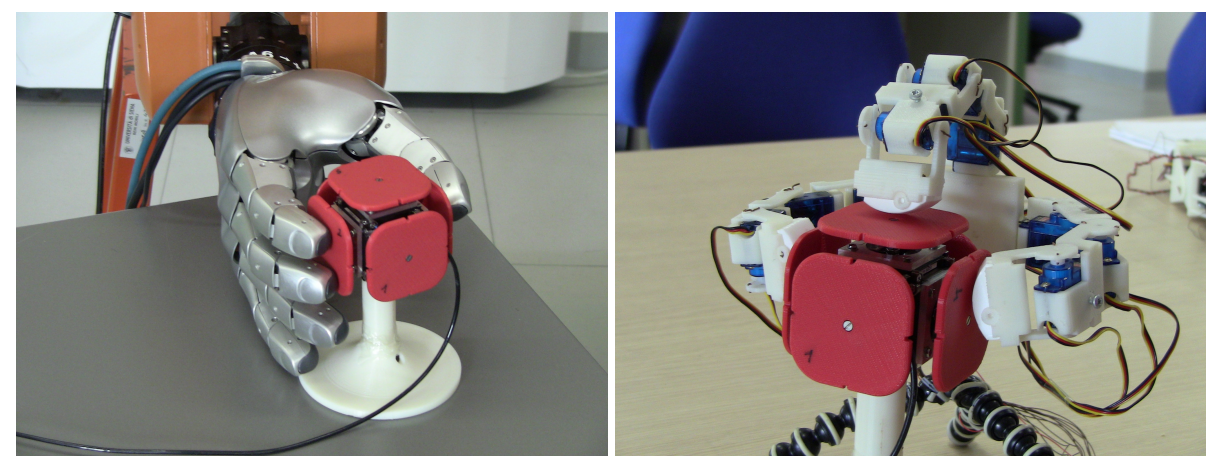

Fig. 11. The two robotic hands grasping the sensorized object in the reference positions.

Table 4. Energy variation error for the DLR-HIT II Hand - Cubic and spherical object

\begin{tabular}{|l|c|c|}
\hline Synergies & \% Error Cubic Obj. & \% Error Spherical Obj. \\
\hline \hline Syn 1 & $1 \%$ & $1 \%$ \\
Syn 2 & $26 \%$ & $22 \%$ \\
Syn 3 & $10 \%$ & $8 \%$ \\
\hline
\end{tabular}

can not be reproduced on the ModHa 39p because there are not adduction/abduction joints on that hand and thus also the total energy variation can not be reproduced.

\section{Conclusions}

The chance of a large diffusion of robotic hands, especially in industrial scenarios, necessarily passes through a simplification of the rules underlying their control laws. In several fields the replacement of well known instruments with new user-friendly and easy-to-control ones has allowed the gaining of new market segments. Close to the simplicity concept, the device independent property is of fundamental importance 


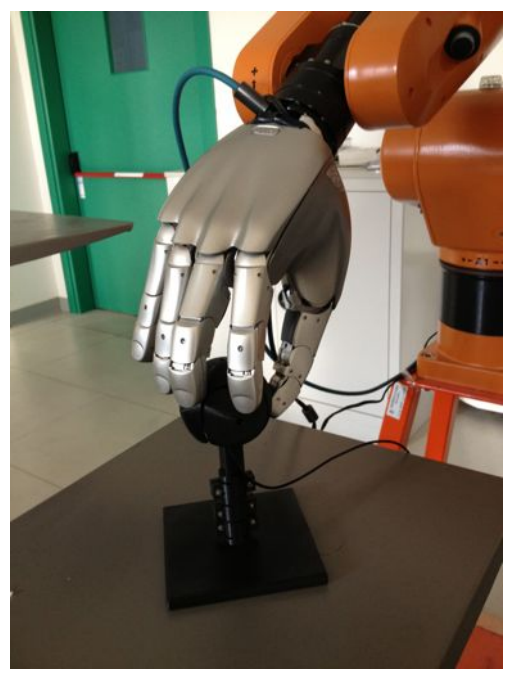

Fig. 12. The DLR-HIT II hand grasping the instrumented object in the sphere configuration

for a wide integration in the hyper-flexible cell scenario. The middleware approach we developed, will ensure the possibility to perform our control approach, based on synergies, upon different existing robot hands.

This is the target of the HANDS.DVI experiment: hiding to users both the complexity of the robotic hand structure, and making possible the use of different devices with the same abstraction layer, i.e. transparent to programmers. While the quest for a solution to the decades-long problem of trading off simplicity and performance in the programming and control of robot hands has often been solved via empirical methodologies, our goal was replicating in the robotic device an organized set of synergies, ordered by increasing complexity, so that a correspondence can be made between any specified task set (in terms of a number of different grasps and exploration actions) and the least number of synergies whose aggregation makes the task feasible. Such "principled simplification" approach will eventually lead to the possibility of expanding tasks in a basis of synergies correctly defined for the artificial hand to be controlled, thus making it possible to come closer to practical applications of such devices in the industrial hyper-flexible cell scenario.

The main innovation of HANDS.DVI concerns the integration of the methodologies and technologies from neuroscience, robotics and control theory in a coherent set of theoretical tools and a methodology for the integration of the current hands model in a larger context such that of hyper-flexible cells. In particular, possible innovations based on the outcome of this project are:

- to allow robot hands to be programmed more easily and to adapt more robustly to different task/environment conditions;

- to improve the human-robot cooperation inside an industrial assembly chain by exploiting similarities between human and robotic hand behaviours. 
Consider as example an operator that has to "teach" to a complex robotic hand the grasp of a new object. In the HANDS.DVI scenario, the operator uses only few knobs (for instance those controlling the first two synergies) to shape the hand around the object decreasing the time and the complexity of the operation. Dually, when virtual artificial hands work as avatars in visuo-haptic rendering of the hyper-flexible cell scenario, exploiting synergies allows to use less sensors to track accurately and in real-time the "principal motion" while performing a manipulation task.

In conclusion, two different experiments to validate the proposed framework were described in this chapter. In the first one, we evaluated the trajectory of a grasped object moved both from the paradigmatic and the robotic hand. We observed that using our mapping method it is possible to reproduce the synergistic movement of a model of the human hand. We further observed that, as imagined, performance decreases according to the limitation of the kinematic structure of the hand. Anyway the generality of the method is preserved. In the second experiment we evaluated the forces exerted by the paradigmatic and the robotic hands over a grasped object. An instrumented object developed within the project has been used to measure these forces. We used an estimation of the total energy used in the grasp to overcame mismatching in the number of contact points that do not consent a direct comparison. Also in this case, our mapping results efficient and the performance decrease with simpler hand.

However, this approach presents some drawbacks. The proposed mapping is based on a heuristic approach: we choose to reproduce a part of the hand motion, which practically corresponds to move and squeeze a spherical object. Although squeezing and moving an object explains a wide range of tasks, many other possibilities exist in manipulating objects which are not modelled with this mapping. Work is in progress to generalize the proposed method enriching the possible motions to be reproduced.

Acknowledgments. This work was supported by the EU Project EC FP7-ICT-231143 ECHORD

\section{References}

1. M. Santello, M. Flanders, and J. Soechting, "Postural hand synergies for tool use," The Journal of Neuroscience, vol. 18, pp. 10105-10115, December 1998.

2. M. Santello and S. J. F., "Force synergies for multifingered grasping," Experimental Brain Research, vol. 133, pp. 457-467, August 2000.

3. J. Lin and T. Wu, "Modeling the Constraints of Human Hand Motion," Urbana, vol. 51, no. 61, p. $801,2000$.

4. R. Linscheid, K. AN, and R. GROSS, "Quantitative analysis of the intrinsic muscles of the hand," Clinical anatomy, vol. 4, no. 4, pp. 265-284, 1991.

5. K. Kim, Y. Youm, and W. Chung, "Human kinematic factor for haptic manipulation: The wrist to thumb," in Haptic Interfaces for Virtual Environment and Teleoperator Systems, 2002. HAPTICS 2002. Proceedings. 10th Symposium on, pp. 319-326, 2002.

6. Y. Youm, M. Holden, and K. Dohrmann, "Finger ray ratio study," tech. rep., 1977.

7. E. Chao, Biomechanics of the hand: a basic research study. World Scientific Publishing Company, 1989. 
8. G. Gioioso, G. Salvietti, M. Malvezzi, and D. Prattichizzo, "An object-based approach to map human hand synergies onto robotic hands with dissimilar kinematics," in Robotics: Science and Systems VIII, see the attached paper, Sidney, Australia: The MIT Press, July 2012.

9. G. Gioioso, G. Salvietti, M. Malvezzi, and D. Prattichizzo, "Mapping synergies from human to robotic hands with dissimilar kinematics: an approach in the object domain," IEEE Trans. on Robotics, 2013.

10. M. Ciocarlie, C. Goldfeder, and P. Allen, "Dimensionality reduction for hand-independent dexterous robotic grasping," in Intelligent Robots and Systems, 2007. IROS 2007. IEEE/RSJ International Conference on, pp. 3270-3275, 2007.

11. A. Peer, S. Einenkel, and M. Buss, "Multi-fingered telemanipulation - mapping of a human hand to a three finger gripper," in Robot and Human Interactive Communication, 2008. ROMAN 2008. The 17th IEEE International Symposium on, pp. 465-470, August 2008.

12. J. Butterfass, M. Grebenstein, H. Liu, and G. Hirzinger, "DLR-hand II: next generation of a dextrous robot hand," in Robotics and Automation, 2001. Proceedings 2001 ICRA. IEEE International Conference on, vol. 1, pp. 109-114, 2001.

13. A. library for Augmented Reality. [Online]: http://www.hitl.washington.edu/ artoolkit/.

14. H. Kato and M. Billinghurst, "Marker Tracking and HMD Calibration for a Video-Based Augmented Reality Conferencing System," in Proc. IEEE ACM Int. Workshop on Augmented Reality, 1999.

15. Y. Ma, S. Soatto, J. Kosecká, and S. Sastry, An Invitation to 3-D Vision: From Images to Geometric Models. Interdisciplinary Applied Mathematics, Springer, 2003.

16. R. Hartley and A. Zisserman, Multiple View Geometry in Computer Vision. Cambridge University Press, 2nd ed., 2004.

17. M. Gabiccini, A. Bicchi, D. Prattichizzo, and M. Malvezzi, "On the role of hand synergies in the optimal choice of grasping forces," Autonomous Robots, Springer, vol. 31, pp. 235-252, 2011.

18. A. Bicchi, J. K. Salisbury, and D. L. Brock, "Contact sensing from force measurements," The International Journal of Robotics Research, vol. 12, no. 3, pp. 249-262, 1993.

19. A. Bicchi, "On the closure properties of robotic grasping," The Int. J. of Robotics Research, vol. 14, no. 4, pp. 319-334, 1995.

20. M. Malvezzi, G. Gioioso, G. Salvietti, D. Prattichizzo, and A. Bicchi, "Syngrasp: a matlab toolbox for grasp analysis of human and robotic hands," in Proc. IEEE Int. Conf. on Robotics and Automation, 2013. 